\title{
Body sensor network-based spasticity detection
}

\author{
Berno J.E. Misgeld, Markus Lueken, Saim Kim and Steffen Leonhardt \\ Philips Chair for Medical Information Technology \\ RWTH Aachen University, 52074 Aachen, Germany, Email: misgeld@hia.rwth-aachen.de
}

\begin{abstract}
Spasticity is a common disorder of the skeletal muscle with a high incidence in industrialised countries, occurring for example after multiple sclerosis or stroke and is associated with brain or spinal cord damage. A quantitative measure of spasticity using body-worn sensors is desirable in order to assess rehabilitative motor training and prevent damage, induced by the externally applied forces or torques from the movement support system. In this contribution we present a new approach to spasticity detection using the Integrated Posture and Activity NEtwork by Medit Aachen (IPANEMA) body sensor network (BSN). Towards this goal, a new electromyography (EMG)-sensor node is developed and employed in human locomotion. Following an analysis of clinical walking data of hemiplegic patients, a novel algorithm is developed based on the idea to detect co-activation of antagonistic muscle groups as observed in the exaggerated stretch reflex with associated limb or joint rigidity. The algorithm is subsequently employed in real walking tests conducted with the IPANEMA BSN showing good detection performance. We furthermore introduce a measure for the spasticity severity based on energy considerations of the recorded EMG signals.
\end{abstract}

Keywords-EMG, neurological diagnostics, spasticity, signal processing

\section{INTRODUCTION}

Electromyography (EMG) has gained importance in a number of areas over the last decades. Besides applications such as neurologic diagnostics and movement analysis, EMG is also increasingly used in training or rehabilitation scenarios in order to provide for example visual or audio feedback. The combination of EMG sensors with inertial measurement devices (IMU) in body sensor networks (BSN) leads to promising solutions towards a wearable evaluation for the human posture control system (PCS) with numerous application areas [1]. However, such systems, although increasingly applied to quantitative assessment of the PCS and associated disorders, are often limited to a research environment [2]. The rationale is the increased complexity of these methods, leading to an increase in instrumentation and personnel costs. Thus small and cheap, yet quality sensitive devices with minimal instrumentation effort are needed to reduce troublesome preparation times for patients and clinicians. Furthermore, such a wearable system is needed to contribute to a clinical diagnostic method remaining easy and practical. Currently developed platforms, presented for example in [3], [4], [5], are promising approaches to such wearable solutions.

A current challenge in the management and the assessment of medical therapy and rehabilitation training is the quantitative evaluation of spasticity, associated with the upper motor neuron syndrome (UMNS). Spasticity was defined by Lance [6] as a motor disorder characterised by a velocity-dependent increase in tonic stretch reflexes ("muscle tonus") with exaggerated tendon jerk, resulting in hyper excitability of the neuron syndrome. Spasticity may occur after spinal cord or brain damage with a high incidence in multiple sclerosis $(85 \%$ of subjects) and stroke (35\% of subjects) [7]. Thus improving the quality of life of the affected subjects by a reduction of spasticity should be the goal of rehabilitation therapy and can be achieved by for example robot-mediated sensorimotor training [8]. A body-worn measurement technique is thereby suggested to enhance the rehabilitation process of UMNS patients suffering from spasticity by providing a quantification of therapy improvement on the one hand and an additional online available spasticity information and associated joint rigidity on the other hand.

Besides qualitative measures of spasticity, like the Ashworth Scale or the Modified Ashworth Scale (MAS) [9], a number of quantitative measures have been introduced by different authors. Among the available techniques are electrophysiological measures, involving EMG and electrical or biomechanical stimulation. Tests based on EMG data recording and electrical stimulation are for example the Hoffman reflex and F-Wave [10] or the tendon reflex and stretch reflex for biomechanical stimulation [11]. In the case of biomechanical measures, continuous EMG recordings are combined with external applied devices or measurement systems to measure forces, positions and velocities [12].

In contrast to these approaches, we propose a method for the online detection of spasticity during normal walking. Our approach is based on the analysis of hemiplegic patient gait data recordings, including EMG-signals from the lower extremities. Our findings show a strong indication of antagonistic muscle co-activation during pathological gait; a pattern occurring at certain gait instances that is typically not observed in normal horizontal walking [13]. Based on these findings, our developed algorithm uses a cross correlation function (CCF) on the filtered and pre-processed EMG-data of antagonistically working muscle groups. The detection algorithm is extended by a weighting of successful detection events with the energy of the corresponding moving window.

This contribution is organised as follows. Section II introduces two new hardware design approaches for the new "Integrated Posture and Activity NEtwork by Medit Aachen" (IPANEMA) BSN EMG sensor node and provides the analysis of hemiplegic gait data. Section III describes the new algorithm, which is then first validated on clinical data followed by a test with experimental data of the IPANEMA BSN EMG node in section IV. Finally, section V ends with a discussion and conclusion.

\section{MATERIALS AND METHODS}

\section{A. IPANEMA BSN}

The "Integrated Posture and Activity NEtwork by Medit Aachen" (IPANEMA) is a modular structure BSN, consisting of a master and multiple slave nodes and allows for easy integration of sensor modules. Figure 1 shows an overview of 
the IPANEMA BSN hardware. Communication between BSN nodes is realised via the $433 \mathrm{MHz}$ industrial, scientific and medical (ISM) band (CC1101 Texas Instruments Inc, USA). Although achieving a lower data rate, IPANEMA is thus less prone to electromagnetic shadowing effects of the human body [5]. The maximum data rate of the $\mathrm{BSN}$ is $250 \mathrm{kbps}$ which is sufficient for the typical activity and physiological parameter measurements, but limits the application to a number of EMG-channels. Communications over the star-shaped network architecture is realised by Time Division Multiple Access (TDMA). IPANEMA hardware and software are designed in a modular way to guarantee communication of various extension boards, like for example the Hardware Abstraction Layers (HAL) and the Medium Access Control (MAC), respectively. The IPANEMA slave nodes can be easily attached to different segments of the human body to provide EMG measurements. Besides the wireless transceiver, shown in Fig. 1, the BSN is equipped with a microcontroller (MSP430F1611, Texas Instruments Inc., USA), two extension ports (CLP160-02-X-D, Samtec USA) and the power management (LTC3558, Linear Technology, USA). The current IPANEMA BSN, which was used for the measurements, is in generation 2.5.

\section{B. EMG extension board}

Two different EMG-sensor nodes (SN) were developed and compared to each other. The first board uses an integrated frond-end with eight separate 24 bit AD-converters (ADS1298, Texas Instruments Inc., USA). The device is specifically designed for electrocardiography (ECG) devices and thus satisfies typical requirements, necessary for biopotential measurements. Besides differential measurement inputs, low noise and a high common mode rejection ratio, the ADS1298 is equipped with ECG specific functionality, like for example pace detection, which were not used in this work. Components, which increase the EMG signal quality, like the right leg drive

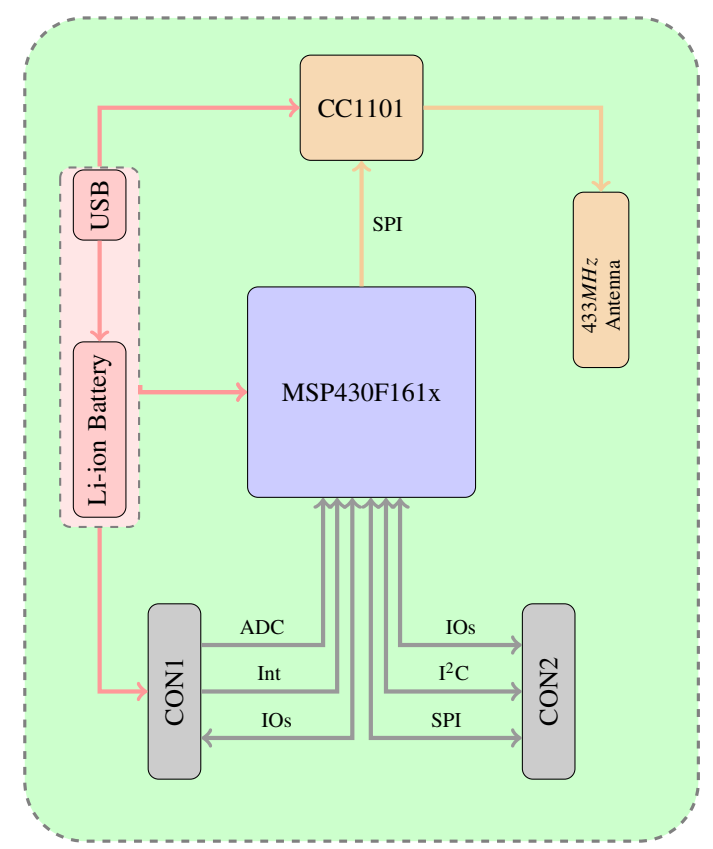

Fig. 1: Design of the IPANEMA main board.
(RLD), are used for this work. Another advantage of the ADS1298 are the programmable gain amplifiers, which can be adjusted by software to a gain ranging from 1 to 12 . In addition to the ADS1298, a second EMG extension board was built from discrete elements. Table I shows the properties of EMG ADS1298 and EMG discrete extension board. The main component of the EMG discrete board is thereby the an analogue front end (MSP430AFE253, Texas Instruments Inc., USA) with integrated microcontroller and three separate 24 bit $\Sigma \Delta$-AD-converters. An instrumentation amplifier (INA128,

TABLE I: Comparison of the developed EMG ADS1298 with the EMG discrete extension board.

\begin{tabular}{|c|c|c|}
\hline Property & EMG ADS1298 & EMG discrete \\
\hline ADC resolution & $24 \mathrm{bit}$ & $24 \mathrm{bit}$ \\
\hline dimensions & $33 \mathrm{~mm} \times 36.5 \mathrm{~mm}$ & $35 \mathrm{~mm} \times 36.5 \mathrm{~mm}$ \\
\hline channels / board & 2 & 1 \\
\hline channel density / area & $0.16 / \mathrm{cm}^{2}$ & $0.078 / \mathrm{cm}^{2}$ \\
\hline max. \# of channels & 8 & 3 \\
\hline max. channels / area & $0.664 / \mathrm{cm}^{2}$ & $0.235 / \mathrm{cm}^{2}$ \\
\hline current drain of the SN & $0.46 \mathrm{~mA}$ & $1,7 \mathrm{~mA}$ \\
\hline input CMRR & $98-115 \mathrm{~dB}$ & $95-105 \mathrm{~dB}$ \\
\hline
\end{tabular}

Texas Instruments Inc., USA) and two second order filter stages, which limit the signal to $20-450 \mathrm{~Hz}$ form the input stage of this module. Furthermore, an additional operational amplifier is used (OPA2131, Texas Instruments Inc., USA) as a right leg drive. The communication with the IPANEMA main board (Fig. 1) is established via the SPI-bus (for both, EMGADS1298 and EMG-discrete boards).

Validation of the two boards using EMG biopotential measurements shows the superior data quality of the ADS1298 extension board. In addition, the absolute value of energy consumption is given by $0.75 \mathrm{~mW} / \mathrm{channel}$ and thus the range the energy consumption of the MSP430F16xx. The discrete extension board needs a much higher supply current. A further advantage of the ADS1298 is the online adaption of gain ratios for each channel, which can be used, for example to adapt the EMG measurement according to a range of conditions. A higher flexibility could for example be obtained by adjusting the gain for a difficult measurement involving obesity. As the result, the decision was made for the ADS1298 extension board, which is able to provide two EMG-channels per extension board each of which sampled at a rate of $1 \mathrm{kHz}$. The signal processing of the EMG ADS1298 extension board is shown in Fig. 2. Here, the left hand side of the figure shows the analogue-digital converter (ADC), including a differential low-pass filter $\left(\mathrm{LP}_{\text {diff }}\right)$, a programmable gain array and a digital decimiser $\mathrm{LP}_{D D}$. The digital signal processing implemented on the computer system, limits the EMG-signal to main spectral components ranging from $20-450 \mathrm{~Hz}$ [14]. This additional computer system receives the EMG-node data from the BSN master via Bluetooth. Thus, two finite impulse response (FIR) filters were employed, as a low pass and a high pass filter with cut-off frequency of $f_{c, H P}=18 \mathrm{~Hz}$ and $f_{c, L P}=450 \mathrm{~Hz}$, respectively. Furthermore, the signal processing chain is extended by a notch filter to reduce the influence of $50 \mathrm{~Hz}$ commercial power frequency.

\footnotetext{
${ }^{1}$ Assuming unaltered dimensions.

${ }^{2}$ Extraction from data sheets.
} 


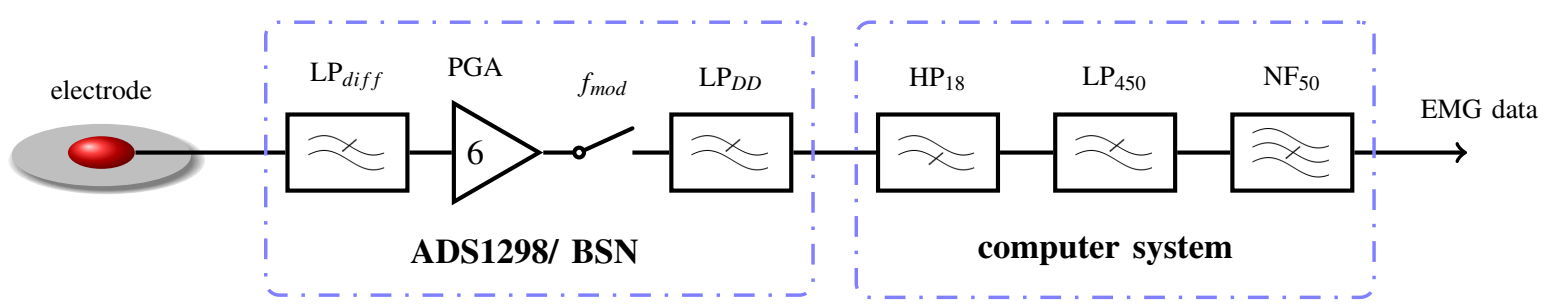

Fig. 2: EMG digital signal processing with LP: low-pass filter, HP: high-pass filter, NF: notch filter, LP $_{\text {diff }}$ : differential low-pass filter, $\mathrm{LP}_{D D}$ : digitial decimiser, PGA: programmable gain array and $f_{\text {mod }}$ : modulation frequency of the ADC (ADS1298/BSN denotes processing on the BSN, where computer system denotes offline data processing on a laptop).

\section{Analysis of hemiplegic gait data}

In order to identify features for the classification of spasticity during normal horizontal ground walking, we analysed EMG data of a patients with dexter spastic hemiparesis. Since spasticity is characterised by an increased velocity-dependent tonic stretch reflex, motion is hindered by a likewise increased velocity dependent stretch resistance of the corresponding muscles. Thus, flexor and stretcher, working as agonist and antagonist in different phases of the gait cycle, stiffen the affected joint. This effect is due to the passive motion of the corresponding muscle in the non-activated cycle of the gait. In case of the lower extremities, spastic hemiparesis usually establishes an outer rotation of the leg in addition to a lifting of the pelvis at the hemiparetic side. An increased stretch of the hip and knee, an inversion of the foot and plantar flexion of the toes can also be observed, whereupon the latter features are usually collectively combined as club foot. Typical characteristics, like the stiffening of the lower extremity and the club foot, could be identified via combined EMG and video analysis of patient data. Using the fact of an involuntarily increase of muscle activity while passively stretched, we considered the activity cycle of antagonistically working muscles for stance. As described in [15], the increase in muscle tonus caused by excitation or strain can also be observed in a healthy person. However, usually this increase decays at a much faster rate. Furthermore the multiple repetition of a single movement pattern does not lead to such a disproportionate increase in muscle tonus like in the spastic hemiparesis case.

A basic consideration for the online detection of spasticity in normal ground level walking was derived from muscle sequence analysis during stance [13]. Fig. 3 shows the typical muscle activation cycle of the lower leg muscle Tibialis Anterior (TA, front tibia) and of the Gastrocnemius Lateralis (GL, inner calf). It can be clearly seen that there is no co-activation

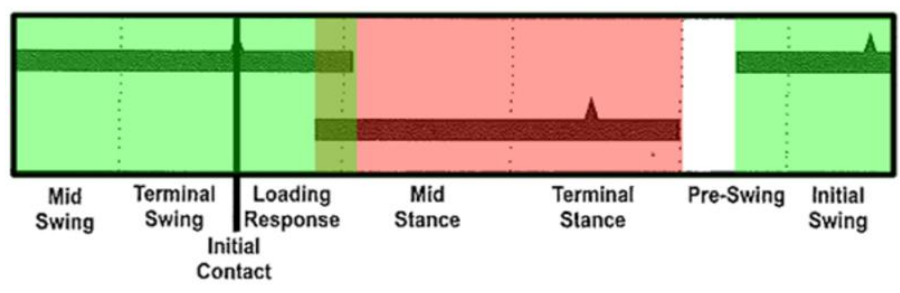

Fig. 3: Muscle activation (y-axis) sequence over a gait cycle (x-axis) during normal horizontal walking for Anterior Tibialis (green) and Gastrocnemius (magenta) (modified from [13]). of these muscles, which was used the data analysis. For that, the recorded EMG-data were rectified and smoothed with a root mean square (RMS) function, working on a $250 \mathrm{~ms}$ timewindow. A detailed data analysis of different muscles showed noticeable changes in the hemiparetic leg, if compared to the healthy leg. Changes are especially noticable when comparing the TA and GL EMG-recorded and filtered data. Figure 4 shows a result of the analysis with filtered data of the TA and GL plotted over a number of gait cycles. It can be clearly seen that a co-activation of the antagonistically working TA and GL muscles occurs in the spastic hemiparetic leg, while in the healthy leg the activation occurs according to the sequence in Fig. 3. Furthermore, the maximum activation of TA and GL takes place at the same time. Based on these findings we develop the algorithm for the online detection of spasticity.

\section{Spasticity DETECTION ALGORITHM}

The main limitation of the algorithm is the later implementation in a low-power application. The spasticity detection and quantification algorithm should hence be developed to provide minimal calculation cost, yet offering a quality sensitive solution to the given problem. Towards this goal, two valid assumptions about the recorded EMG-signals were made. On the one hand, the EMG-signal can be seen as a stationary random process, if we choose a time-window that is small enough. On the other hand, it is assumed that the statistical characteristics of two stationary EMG-random processes are independent, yet time-delays occur mutually for both processes. Therefore, we are able to introduce the first function for peak detection (PD) with the assumption of

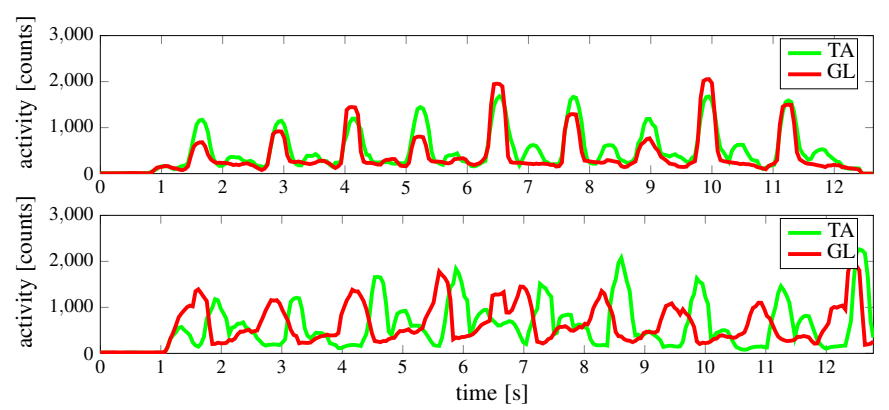

Fig. 4: EMG activity recordings of hemiplegic gait data; pathologic right leg (upper) and healthy left leg (lower) comparing data of the muscles Tibialis Anterior (TA) and Gastrocnemius Lateralis (GA). 
stationary processes. Consider two windows of length $N$ for data vectors $s_{T A}(n)$ and $s_{G L}(n)$ at time-step $k$ for which the cross-correlation function (CCF) $R_{T A, G L}(m)$ is defined as

$$
R_{T A, G L}(m)= \begin{cases}\sum_{n=0}^{N-m-1} s_{T A}(n+m) s_{G L}(n), & m \geq 0 \\ R_{T A, G L}(-m), & m<0,\end{cases}
$$

with positive integers $n, m$ is the continuous index and $c(m)$ denotes the cross-correlation vector of length $2 N-1$ given by

$$
c(m)=R_{T A, G L}(m-N) .
$$

Based on the middle of the $\mathrm{CCF} c_{N}$, the nearest maximum in a neighborhood $\Delta n$ is determined. If the nearest neighbourhood maximum is the global maximum over the time window, a coactivation is assumed to be detected and might be due to spastic co-contraction. The function thus gives a binary information over the gait cycle $K_{C A} \in\{0,1\}$. Fig. 5 shows the CCF the healthy (left) and the spastic hemiparetic lower limb EMG signals of $s_{T A}$ and $s_{G L}$ over two windows of length $N=500$. Normalised CCF varies strongly for the two different legs, as can be clearly seen from the lower part of Fig. 5. Thereby the lower right subplot indicates an occurrence of antagonist muscle co-contraction and whereas the lower left subplot does not.

In addition to the detection of antagonistic muscle cocontraction due to spasticity, the case of muscular inactivity of both muscles must be excluded from a possible detection. For that, the binary detection value of the current time-window was weighted by the signal energy equivalent, given by the square of the 2-norm

$$
E_{i}=\left\|s_{i}\right\|_{2}^{2}=\left\langle s_{i}, s_{i}\right\rangle, \quad i \in\{T A, G L\} .
$$

In addition to that, the algorithm is continuously updated with the maximum of the signal energy which is then used to normalise newly calculated energy content. Thus changing
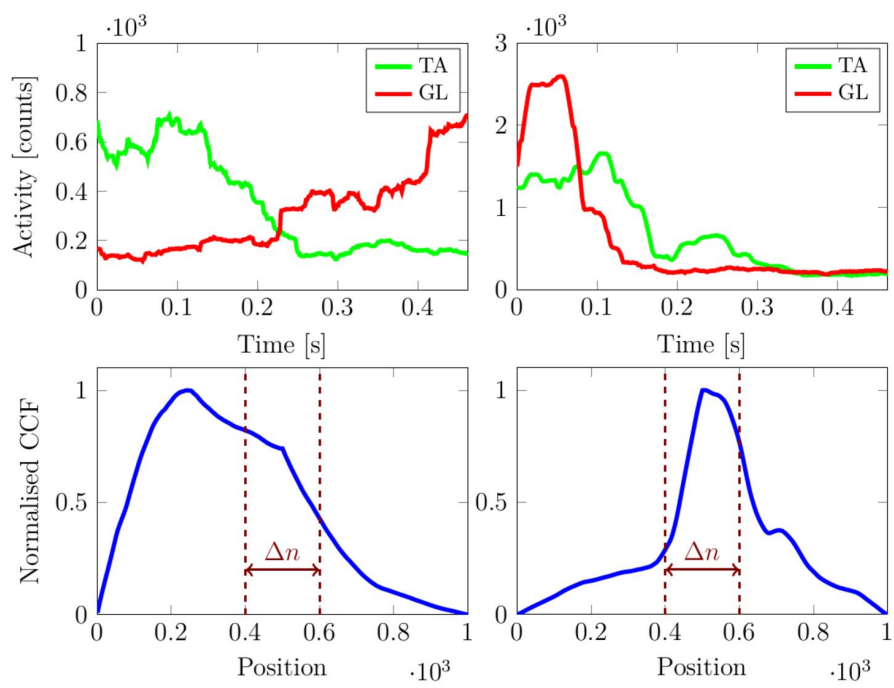

Fig. 5: Rectified, average mean EMG signal of TA and GL for 500 values (upper part, different y-axis ranges) and resulting CCF (lower part) of healthy (left column) and spastic hemiparetic limb (right).

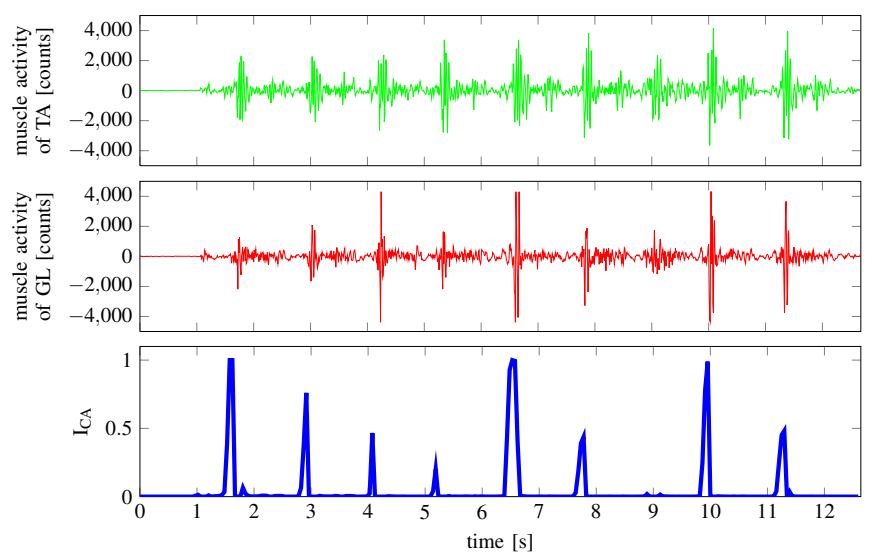

Fig. 6: Input EMG-signals and algorithm output for pathologic gait pattern (negative counts result from signed integers).

measurement conditions or muscle fatigue are managed, avoiding a normalisation of the EMG-signal at the beginning of a measurement. The spasticity index is given by weighting of the decision variable $K_{C A}$

$$
I_{C A}=K_{C A} E_{T A} E_{G L},
$$

with the energy equivalent of the corresponding muscles. In a heuristic procedure, parameters size of time-window $N$, maximum distance to mid-CCF $\Delta n$ and relative increment for the new time-window $s_{r e l}$ were tuned. $N$ and $s_{r e l}$ are critical for fast execution of the algorithm, however too small values of $N$ and $s_{\text {rel }}$ result in a smaller distance to mid-CCF and a time-lag of subsequent detections, respectively. Thus parameters were set to $N=200$ and $s_{r e l}=0.25$. The maximum distance to mid$\mathrm{CCF}$ was set to $\Delta n=0.1$ following experimental results, which allows for a relative deflection of $10 \%$. Fig. 6 shows the result of the algorithm for spastic hemiparetic side of the patient. The algorithm strongly indicates co-contraction of antagonistically working muscles due to spasticity. In the case of the healthy leg (not shown), detection values $I_{C A}$ are below 5\%, except for an initial phase, where walking is started and values of about $50 \%$ could be observed.

\section{EXPERIMENTAL VALIDATION}

After validation of the algorithm with clinical data, the algorithm was experimentally validated with the new IPANEMA BSN EMG node. Walking tests were conducted with surface EMG (sEMG) Ag/AgCl electrodes (2228, 3M, St. Paul, USA) following standard procedures for skin preparation. Two sEMG electrodes were applied to the TA and GL and the RLD reference electrode was placed at the ankle. EMG tests were conducted on horizontal ground walking, in which a healthy subject was instructed to mimic the movement pattern of spastic hemiparetic gait following a detailed video analysis of the clinical data. Since the club foot was evidently manifested in the recorded clinical data, it was adopted as one of the main characteristics for walking. However, it has to be noted that measurement results cannot be used to extend physiological understanding of spastic hemiparesis and walking tests were conducted to test the algorithm in connection with the IPANEMA BSN EMG real-time recorded signals. The data was recorded to measure and compare the quality of 
the IPANEMA BSN recordings and the spasticity detection algorithm. At the beginning of an experiment the muscles were co-contracted to about $70 \%$ to get an initial value for the normalisation of the signal. The result of the club foot simulated gait is shown in Fig. 7. Co-activation of muscles during gait was successfully detected and subsequently weighted by agonist and antagonist energy. In case of normal horizontal ground walking (not shown), besides an initial co-activation (for initial normalisation) the algorithm did not detect any coactivation of TA and GL and $I_{C A}$ remained below 5\%. Peak values of $E_{C A}$ during the mimicked club foot walking, apart from the initial co-activation phase, showed values of up to $65 \%$ (where in the initial co-activation phase an $I_{C A}$ of about $70 \%$ was achieved).

\section{CONCLUSION AND DISCUSSSION}

In this contribution, we described the development of two IPANEMA BSN - EMG extension boards, whose intended use was online spasticity detection in hemiparetic walking, but are not limited to this application. The advantage was given to the ADS1298 board because of the low power consumption and the higher number of available channels. The new IPANEMA BSN extension board is able to support two EMG-data channels with a rate of $1 \mathrm{kHz}$ each, which is enough for the typical EMG application. In a detailed frequency analysis of clinical hemiparetic walking EMG data, we were not able to identify significant characteristics which could be used to distinguish between spastic hemiparetic and healthy walking. We thus developed a novel algorithm for spasticity detection, based on the idea to detect co-activation of antagonistically working muscles and extended the index by an energy weighting of the corresponding muscles. Initial evaluation using clinically recorded data of spastic hemiparetic walking revealed good performance with high incidence of pathological gait patterns, compared to a low incidence of the healthy leg of the patient. However, the available data basis is not sufficient to conclude the proposed index $I_{C A}$ as a general classifier for spasticity in other manifestations. Thus, future work will be concerned with the extension of the EMG-data basis to include test subjects of varying degree of spasticity in different manifestations. Furthermore, the assessment of the identified features by a supervised learning classifier, like for

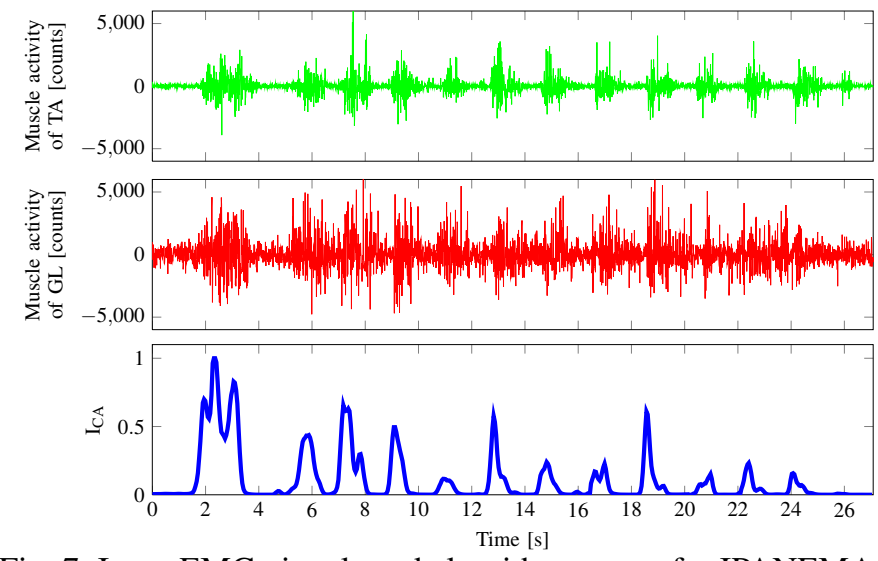

Fig. 7: Input EMG-signals and algorithm output for IPANEMA BSN EMG mimicked hemiparetic walking. example a support vector machine is another option for future work. However, one has to bear in mind the limited amount of calculation power. Finally, we conclude that the index $I_{C A}$ based on the co-activation of the Tibialis Anterior and the Gastrocnemius Lateralis can be used to specify spasticity in hemiparetic walking. A cross correlation function, extended by sEMG signal energy weightings enables the computation of an online spasticity quantification index, which shows promising results for clinical as well as IPANEMA BSN EMG data.

\section{ACKNOWLEDGMENT}

The authors express their sincere gratitude to to the Department of Orthopedics and Trauma Surgery, Heidelberg University Hospital, Germany for providing gait data.

\section{REFERENCES}

[1] H. Harms, O. Amft, R. Winkler, J. Schumm, M. Kusserow, and G. Troester, "Ethos: Miniature orientation sensor for wearable human motion analysis," in Sensors, 2010 IEEE, Nov 2010, pp. 1037-1042.

[2] J. Pons and D. Torricelli, Eds., Emerging Therapies in Neurorehabilitation. Springer Verlag Heidelberg, 2014.

[3] C. Ho, J. Evans, M. Mark, J. Rabaey, M. Koplow, L. Miller, A. Chen, E. Reilly, and P. Wright, "Technologies for an autonomous wireless home healthcare system," in BSN - Sixth International Workshop on, June 2009, pp. 29-34.

[4] Z. Zhang, C. Wong, and G.-Z. Yang, "Forearm functional movement recognition using spare channel surface electromyography," in Body Sensor Networks (BSN), 2013 IEEE International Conference on, May 2013, pp. 1-6.

[5] S. Kim, C. Brendle, H.-Y. Lee, M. Walter, S. Gloeggler, and S. Leonhardt, "Evaluation of a $433 \mathrm{mhz}$ band body sensor network for biomedical applications," Sensors, vol. 13(1), pp. 898-917, 2013.

[6] J. Lance, Spasticity: Disordered Motor Control, R. Feldman, R. Young, and W. Koella, Eds. Year Book Medical Publishers, 1980.

[7] D. K. Sommerfeld, E. U.-B. Eek, A.-K. Svensson, L. W. Holmqvist, and M. H. von Arbin, "Spasticity after stroke: Its occurrence and association with motor impairments and activity limitations," Stroke, vol. 35, no. 1, pp. 134-139, 2004.

[8] F. Posteraro, S. Mazzoleni, S. Aliboni, B. Cesqui, A. Battaglia, M. C. Carrozza, P. Dario, and S. Micera, "Upper limb spasticity reduction following active training: A robot-mediated study in patients with chronic hemiparesis," Journal of Rehabilitation Medicine, vol. 42, no. 3, pp. 279-281, 2010.

[9] R. W. Bohannon and M. B. Smith, "Interrater reliability of a modified ashworth scale of muscle spasticity," Physical Therapy, vol. 67, no. 2, pp. 206-207, 1987.

[10] C. Yates, K. Garrison, N. B. Reese, A. Charlesworth, and E. GarciaRill, "Chapter 11-novel mechanism for hyperreflexia and spasticity," Prog Brain Res, vol. 188, pp. 167-80, 2011.

[11] M. Chardon, N. Suresh, and W. Rymer, "An evaluation of passive properties of spastic muscles in hemiparetic stroke survivors," in Engineering in Medicine and Biology Society (EMBC), 2010 Annual International Conference of the IEEE, Aug 2010, pp. 2993-2996.

[12] K. S. Kim, J. H. Seo, and C. G. Song, "Portable measurement system for the objective evaluation of the spasticity of hemiplegic patients based on the tonic stretch reflex threshold," Med Eng Phys, vol. 33, no. 1, pp. $62-69,2011$

[13] J. Perry and J. Burnfield, Gait Analysis: Normal and Pathological Function. Slack Incorporated, 2010.

[14] C. J. D. Luca, L. D. Gilmore, M. Kuznetsov, and S. H. Roy, "Filtering the surface emg signal: Movement artifact and baseline noise contamination," Journal of Biomechanics, vol. 43, no. 8, pp. 1573 - 1579, 2010.

[15] B. Bobath, Adult Hemiplegia: Evaluation and Treatment. ButterworthHeinemann, 1990. 